\title{
Stage IIB Choroidal and Ciliary Body Melanoma AJCC v8
}

National Cancer Institute

\section{Source}

National Cancer Institute. Stage IIB Choroidal and Ciliary Body Melanoma A/CC V8. NCI

Thesaurus. Code C140663.

Stage IIB includes: (T 2b, N0, M0); (T3a, N0, M0). T2b: Tumor size categ ory 2 with ciliary body involvement. T3a: T umor size category 3 without ciliary body involvement and extraocular extension. N0: No regional lymph node metastasis. M0: No distant metastasis. (AJCC 8th ed.) 